\title{
An AC Magnetohydrodynamic Microfluidic Switch
}

A.V. Lemoff, A.P. Lee

This article was submitted to MicroTAS 2000, The Netherlands, May 14-18, 2000

March 2, 2000

U.S. Department of Energy

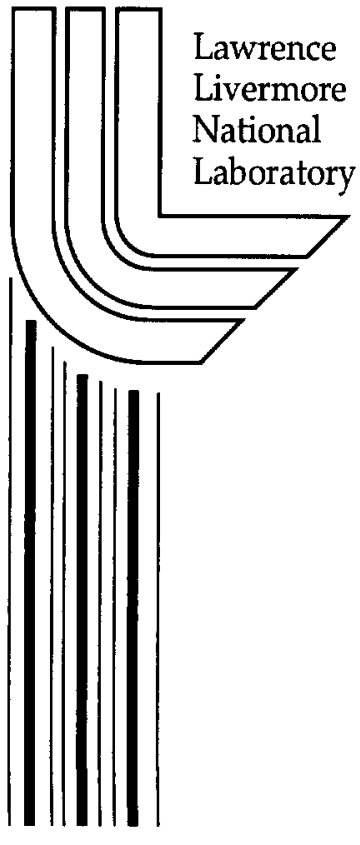




\section{DISCLAIMER}

This document was prepared as an account of work sponsored by an agency of the United States Government. Neither the United States Government nor the University of California nor any of their employees, makes any warranty, express or implied, or assumes any legal liability or responsibility for the accuracy, completeness, or usefulness of any information, apparatus, product, or process disclosed, or represents that its use would not infringe privately owned rights. Reference herein to any specific commercial product, process, or service by trade name, trademark, manufacturer, or otherwise, does not necessarily constitute or imply its endorsement, recommendation, or favoring by the United States Government or the University of California. The views and opinions of authors expressed herein do not necessarily state or reflect those of the United States Government or the University of California, and shall not be used for advertising or product endorsement purposes.

This is a preprint of a paper intended for publication in a journal or proceedings. Since changes may be made before publication, this preprint is made available with the understanding that it will not be cited or reproduced without the permission of the author.

This report has been reproduced directly from the best available copy.

Available electronically at http://www.doe.gov/bridge

Available for a processing fee to U.S. Department of Energy

and its contractors in paper from

U.S. Department of Energy

Office of Scientific and Technical Information

P.O. Box 62

Oak Ridge, TN 37831-0062

Telephone: (865) 576-8401

Facsimile: (865) 576-5728

E-mail: reports@adonis.osti.gov

Available for the sale to the public from

U.S. Department of Commerce

National Technical Information Service

5285 Port Royal Road

Springfield, VA 22161

Telephone: (800) 553-6847

Facsimile: (703) 605-6900

E-mail: orders@ntis.fedworld.gov

Online ordering: http://www.ntis.gov/ordering.htm

OR

Lawrence Livermore National Laboratory

Technical Information Department's Digital Library

http://www.llnl.gov/tid/Library.html 


\title{
AN AC MAGNETOHYDRODYNAMIC MICROFLUIDIC SWITCH
}

\author{
Asuncion V. Lemoff* and Abraham P. Lee \\ Center for Microtechnology, Lawrence Livermore National Laboratory \\ 7000 East Ave. L-223, Livermore, CA 94550 \\ E-mail: lemoff1@linl.gov, lee60@|lnl.gov \\ *also Department of Applied Science, University of California, Davis
}

\begin{abstract}
A microfluidic switch has been demonstrated using an AC Magnetohydrodynamic (MHD) pumping mechanism in which the Lorentz force is used to pump an electrolytic solution. By integrating two AC MHD pumps into different arms of a Y-shaped fluidic circuit, flow can be switched between the two arms. This type of switch can be used to produce complex fluidic routing, which may have multiple applications in $\mu$ TAS.
\end{abstract}

Keywords: magnetohydrodynamic, fluidic switch, Lorentz force, micropump

\section{Introduction}

One microfluidic component that can enable $\mu$ TAS is a microfluidic switch. Microfluidic switching allows for complex routing of a sample in microchannels for possible mixing with different reagents or for routing the sample into different detection systems. These possible uses of a microfluidic switch are illustrated in Fig. 1.

We have developed a new kind of microfluidic switch using an AC Magnetohydrodynamic (MHD) pumping mechanism. An AC Magnetohydrodynamic micropump has previously been presented in which the Lorentz force is used to propel an electrolytic solution along a microchannel etched in silicon [1]. This micropump has no moving parts, produces a continuous (not pulsatile) flow, and is compatible with solutions containing biological specimens. By integrating two AC MHD pumps into different arms of a fluidic Y-channel, flow can be made to switch into or out of either arm.

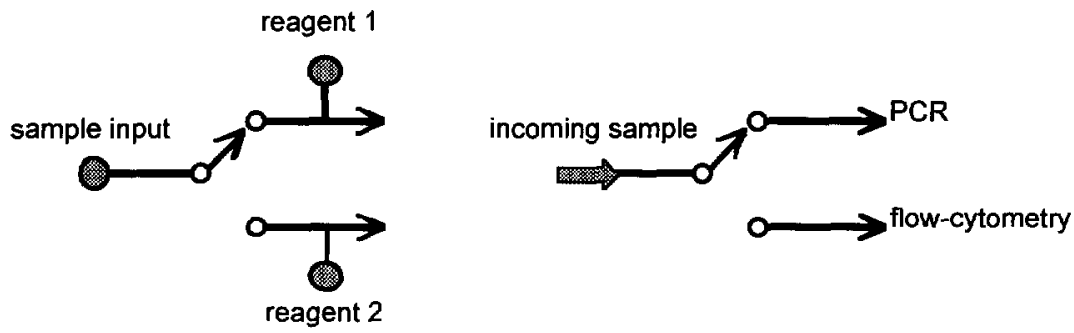

Figure 1. Possible uses for a microfluidic switch. Left. Switch can be used to direct a sample input to react with different reagents. Right. Switch can be used to direct a sample into different detection systems. 


\section{Theory}

In an $\mathrm{AC}$ MHD micropump, a sinusoidal $\mathrm{AC}$ electrical current and a perpendicular, synchronous sinusoidal AC magnetic field pass through an electrolytic solution, transverse to a microchannel. This produces a Lorentz force along the channel, causing the solution to flow. This geometry is illustrated in Fig. 2. The time averaged Lorentz force is given by:

$$
F=I B w \int_{0}^{2 \pi} \sin \omega t \sin (\omega t+\phi) d \omega t
$$

where $I$ is the current amplitude (measured in Amperes) across the microchannel, $B$ the magnetic field amplitude (measured in Tesla), and $w$ the width of the microchannel. From Eq. (1), the ability to control the phase allows for controlling both the flow speed and the flow direction. The integrand can have a value between $-1 / 2$ and $1 / 2$. At $0^{\circ}$, the integrand is positive and corresponds to a flow in one direction. At $180^{\circ}$, the integrand is negative and corresponds to flow in the opposite direction, and at $90^{\circ}$ the integrand is zero corresponding to no flow. Because the Lorentz force varies in magnitude through one period of oscillation, the flow is actually pulsed; however at high frequencies, the pulsed volume is so small that the flow is effectively continuous, reflecting only the time-averaged force.

A microfluidic switch can be constructed by integrating two pumps into the

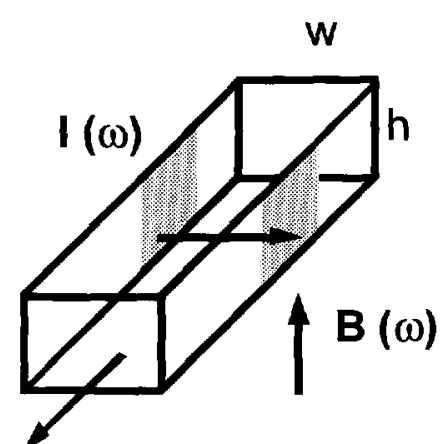

$\mathbf{F}$

Figure 2. Vector-diagram of an AC MHD micropump.

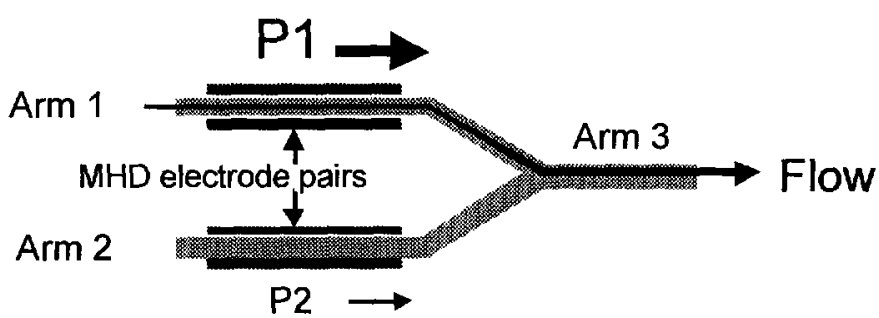

Figure 3. Conceptual diagram of MHD microfluidic switch.

configuration shown in Fig. 3. When only the pump in arm 1 is actuated (for example in the direction towards arm 3), the flow that is produced divides into both arm 2 and arm 3 . In order to stop the flow in arm 2, while flow continues from arm 1 to arm 3 , the pump in arm 2 must be actuated to produce the necessary pressure to cancel the forces in that arm. This pressure will in general be smaller than the pumping pressure in arm 1. If the same electromagnet is used to actuate both pumps, then there are two methods by which the forces in arm 2 can be cancelled. The first method is to adjust the current flowing between the electrodes in arm 2 to the desired fraction of the current flowing in arm 1. The second method is to use equal electrode currents in arm 1 and arm 2 and to adjust the relative phase of the current in arm 2 with respect to the electromagnet. At a particular phase, the forces in arm 2 will be cancelled. Using either method, flow can be switched between the two arms by interchanging the electrode currents between the two arms. Because a change in current amplitude will affect temperature and $\mathrm{pH}$ of the solution, the second method, in which current amplitude is held constant, is preferable. 


\section{Experimental}

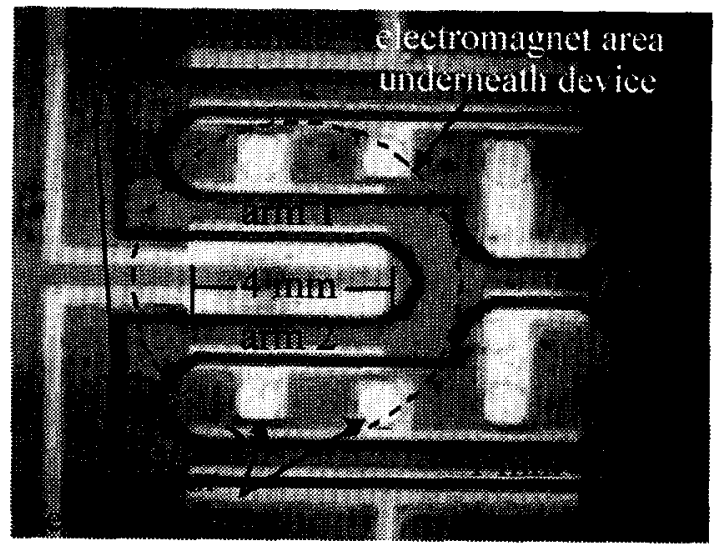

Figure 4. Photograph of fluidic switch. Only two MHD electrode pairs are actuated. Channels are $1 \mathrm{~mm}$ wide and $300 \mu \mathrm{m}$ deep. Electrode length of $4 \mathrm{~mm}$.

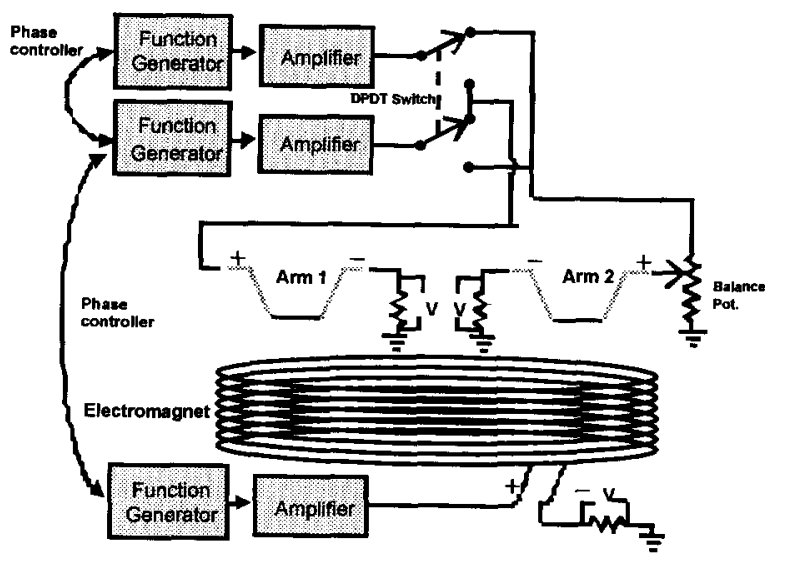

Figure 5. Experimental set-up of fluidic switch..

The devices are fabricated by etching a v-groove $300 \mu \mathrm{m}$ deep and $1 \mathrm{~mm}$ wide on a silicon wafer, depositing metal electrodes on a thin oxide layer that continue down the walls of the groove, and then anodically bonding the wafer to a plate of glass. Holes are drilled in the glass to allow for contacting the electrodes and for fluidic input and output. A photograph of a fluidic circuit in which liquid is switched between two closed loops is shown in Fig. 4. An electromagnet, positioned beneath the device, produces a magnetic field normal to the plane of the wafer. Only the two inner MHD electrode pairs, which are enclosed in the diameter of the electromagnet core, were actuated and switched as indicated in Fig. 4. The other MHD electrode pairs could be actuated with a larger diameter electromagnet core.

The fluidic circuit shown in Fig. 4 was filled with a $1 \mathrm{M} \mathrm{NaCl}$ solution containing $5 \mu \mathrm{m}$ diameter polystyrene beads. The flow velocity was measured in arms 1,2 and 3 by

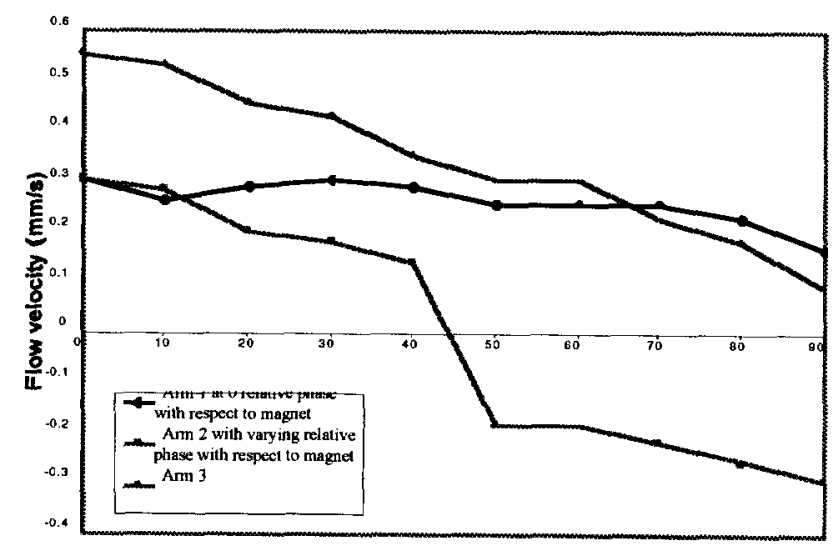

Relative phase of Arm 2 (degrees)

Figure 6. Flow velocities of arms 1, 2, 3 with the relative phase of arm 2 varying with respect to the electromagnet. tracking the motion of the beads using a computer with video capture software. Switching was observed by switching the currents between the two pumps. The experimental set-up is illustrated in Fig. 5. Ideally, with the same electromagnet and the same applied electrode current, both arms should produce the same flow. In reality, the flow can differ due to variations in magnetic field strength between the two arms. To compensate for this, a potentiometer was added in series with one of the arms as shown in Fig. 5. 


\section{Results and discussion}

The fluidic switch was actuated as shown in Fig. 3. Arms 1 and 2 were actuated with the same electrode current. While arm 1 was kept at $0^{\circ}$ relative phase with respect to the electromagnet, the phase of arm 2 was varied to determine the phase required to cancel the flow in that arm. This was found to be approximately $45^{\circ}$ relative phase as shown in Fig. 6. With this phase, flow in arm 2 is completely blocked. By toggling the double-pole double throw switch, shown in Fig. 5, flow can be switched between arm 1 and arm 2 .

Within the resolution of the measurement system, the switching of flow between the two arms is instantaneous. Figure 7 shows three consecutive video frames, captured at the moment of switching, each separated by 0.033 seconds. Between the first two frames, each of the tracked particles moves in the direction of the second arm, as indicated by the arrows in the diagram. Between the second and third frames, all of the particles have moved in the direction of the first arm, indicating that flow switching has already occurred.

\section{Conclusions}

Using one electromagnet, an $\mathrm{AC}$ Magnetohydrodynamic microfluidic switch has been demonstrated by integrating AC MHD micropumps. Microfluidic switching allows for complex routing of a

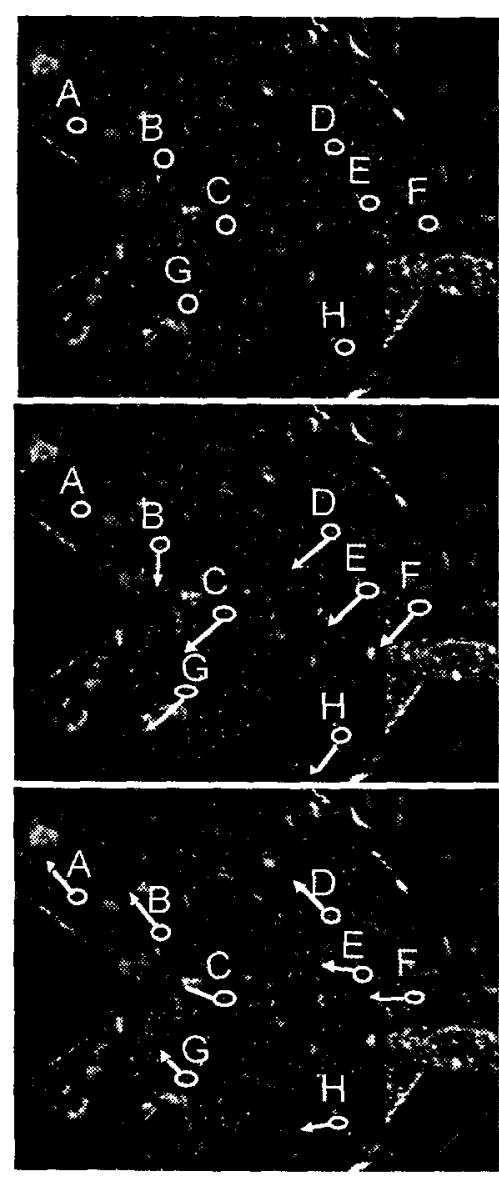

Figure 7. Tracking 8 particles in 3 consecutive frames $\mathbf{0 . 0 3 3}$ seconds apart. Arrows show direction of displacement from the previous frame. sample in microfluidic channels.

\section{Acknowledgments}

The authors would like to acknowledge Robin Miles, Charles McConaghy and Christopher Fuller from the Center for Microtechnology of LLNL for their valuable technical inputs. The authors would also like to acknowledge Dr. Greg Cutler and Dr. Brian Lemoff of Agilent Laboratories for valuable help on electromagnets and help on measurements, respectively. This work was performed under the auspices of the US Department of Energy by the Lawrence Livermore National Laboratory under contract W-7405-ENG-48.

\section{References}

1. A.V. Lemoff, A.P. Lee, R.R. Miles and C.F. McConaghy, "An AC Magnetohydrodynamic Micropump: Towards a True Integrated Microfluidic System", Transducers '99 The $10^{\text {th }}$ International Conference on Solid-State Sensors and Actuators, Sendai, Japan, 7-10 June (1999), p. 1126-1129. 